\title{
Mathematical Analysis on Pulsatile Flow through a Catheterized Stenosed Artery
}

\author{
Shafi Ullah Siddiqui, Chhama Awasthi* \\ Department of Mathematics, Harcourt Butler Technical University, Kanpur, India \\ Email: susiddmath_56@rediffmail.com, *awasthi.chhama@gmail.com
}

How to cite this paper: Siddiqui, S.U. and Awasthi, C. (2017) Mathematical Analysis on Pulsatile Flow through a Catheterized Stenosed Artery. Journal of Applied Mathematics and Physics, 5, 1874-1886. https://doi.org/10.4236/jamp.2017.59157

Received: August 24, 2017

Accepted: September 25, 2017

Published: September 28, 2017

Copyright $\odot 2017$ by authors and Scientific Research Publishing Inc. This work is licensed under the Creative Commons Attribution International License (CC BY 4.0).

http://creativecommons.org/licenses/by/4.0/ c) (i) Open Access

\begin{abstract}
In this paper, the pulsatile flow of blood through an inclined catheterized stenosed artery is analyzed. Perturbation method is used to solve the implicit system of partial differential equations with suitable boundary conditions. Various analytical expressions axial velocity, flow rate, wall shear stress and effective viscosity have been derived with the help of MATLAB for understanding the fluid flow phenomena. The combined effect of catheterization, body acceleration, slip and inclination has been seen by plotting the graph and observed that axial velocity and flow rate increases with the increase in body acceleration, inclination angle and slip velocity while axial velocity diminishes on increasing the catheter radius. Wall shear stress increases with the increase in catheter radius and body acceleration but presence of slip velocity reduces the wall shear stress. Effective viscosity diminishes on increasing body acceleration and inclination angle, whereas slightly augmented in non-inclined stenosed artery.
\end{abstract}

\section{Keywords}

Newtonian Fluid, Blood Flow, Stenosis, Pulsatile Flow, Catheterized Artery

\section{Introduction}

In recent years, the study of blood flow through obstructed arteries has received much attention, due to its ample importance in human cardiovascular system. The theoretical analysis on blood flow is very useful as it plays a significant role to diagnose and understand many cardiovascular diseases such as coronary thrombosis, angina, pectoris, strokes etc. The reason behind the malfunction of cardio-vascular system is the presence of fats, cholesterol and lipoproteins at the sites of atherosclerotic lesion in the artery. By the development of atherosclerotic plaques that protrude into the lumen, arteries get narrowed and stenosed arteries are formed. Presence of atherosclerosis (stenosis), increased the resistance and 
therefore blood flow reduced inside the artery and also the remarkable change occurred in pressure distribution and wall shear stress. Bennett [1] has observed that the presence of wall slip at the flow boundaries decreases the apparent (effective) viscosity. Tu and Deville [2] observed the blood flow in diseased conditions.

Mathematical models for blood flow through stenosed arterial segment, by taking a velocity slip condition at the constricted wall were developed [3] [4]. Our Body experiences the acceleration while running, jumping and travelling etc., due to which different health related issues arise such as headache, loss of vision, increase in pulse rate etc. Sud and Sekhon [5] studied on pulsatile flow of blood through a rigid circular tube subjected to periodic body acceleration considering blood as Newtonian fluid. In [6] [7] [8], the pulsatility of blood is discussed by treating blood as a Newtonian fluid. Liepsch [9] compared the details of flow behaviors with hemodynamic approach and observed that flow rate varies due to pulsatile nature of blood. Since heart pumps of the blood are periodic in nature, the blood flow in narrow arteries can be assumed as pulsatile. Young [10] observed that blood can be assumed as Newtonian when it passes through narrow arteries $(0.02-0.1 \mathrm{~mm})$ at low shear rate $(<10 / \mathrm{s})$ particularly in diseased state while blood being suspension of corpuscles in plasma behaves like a non-Newtonian fluid when it flows through larger arteries at high shear rate [11] [12].

Catheter is a thin hollow flexible tube made from medical grade materials and is used in a broad range of functions. Srinivasacharya and Srikanth [13] observed that catheterization refers to a procedure in which a long, thin, flexible plastic tube (catheter) is inserted into an artery. The insertion of a catheter into a constricted tube (i.e. stenosed artery) results in an annular region between the walls of the catheter and artery. This will alter the flow field, modify the pressure distribution and increase the resistance. MacDonald [14] considered the pulsatile blood flow in a catheterized artery and obtained theoretical estimates for pressure gradient corrections for catheters, which are positioned eccentrically, as well as coaxially with the artery. Karahalios [15] discussed some possible effects of a catheter on the arterial wall.

Cardiac catheterization is a procedure used to diagnose and treat cardiovascular conditions in surgical procedures. During cardiac catheterization, coronary angiography is done by inserting a catheter in an artery or vein in your groin, neck or arm and threaded through your blood vessels to your heart. Back and Denton [16] studied the variation of wall shear stress and discussed its clinical importance in coronary angioplasty. An appropriate size of a catheter is required for each experiment in order to reduce the error due to the wave reflection at the tip of the catheter [17]. Sarkar and Jayaraman [18] discussed the changed flow patterns of pulsatile blood flow in a catheterized stenosed artery. Effect of slip velocity on blood flow through a catheterized stenosed artery is studied in [19].

All above mentioned studies are based on the horizontal blood carrying vessels. Although it is well-known that many ducts in physiological systems are no horizontal, few of them have some inclination to the axis. Therefore, a gravita- 
tional force has been accounted there due to inclination. Chaturani and Upadhyay [20] investigated the gravity flow of fluid with couple stress along an inclined plane. Vajravelu, Sreenadh and Ramesh Babu [21] showed the theory on peristaltic transport of Herschel Bulkley fluid through an inclined tube. The study on pulsatile flow of blood through rigid inclined circular tubes under the influence of periodic body acceleration is proposed in [22]. Maruti Prasad and Radha Krishnamcharya [23] have analyzed a model of steady blood flow through an inclined artery with a stenotic wall. Biswas and Paul [24] developed a mathematical model on the steady flow of blood through an inclined tapered constricted artery assuming blood as Newtonian fluid, with an axial slip velocity at the vessel wall. Pulsatile flow of blood in a catheterized inclined artery [25] with a slip velocity at the stenosed arterial wall under the influence of magnetic field is also discussed. To generalize the problem further, present model has been established to analyze the effect of catheterization, body acceleration, slip velocity and pulsatility on arterial flow characteristics in inclined stenosed artery. The detail study on the present work is discussed through the graph considering blood as Newtonian fluid.

\section{Mathematical Formulation}

Consider the pulsatile flow of blood through an inclined axially symmetric ca

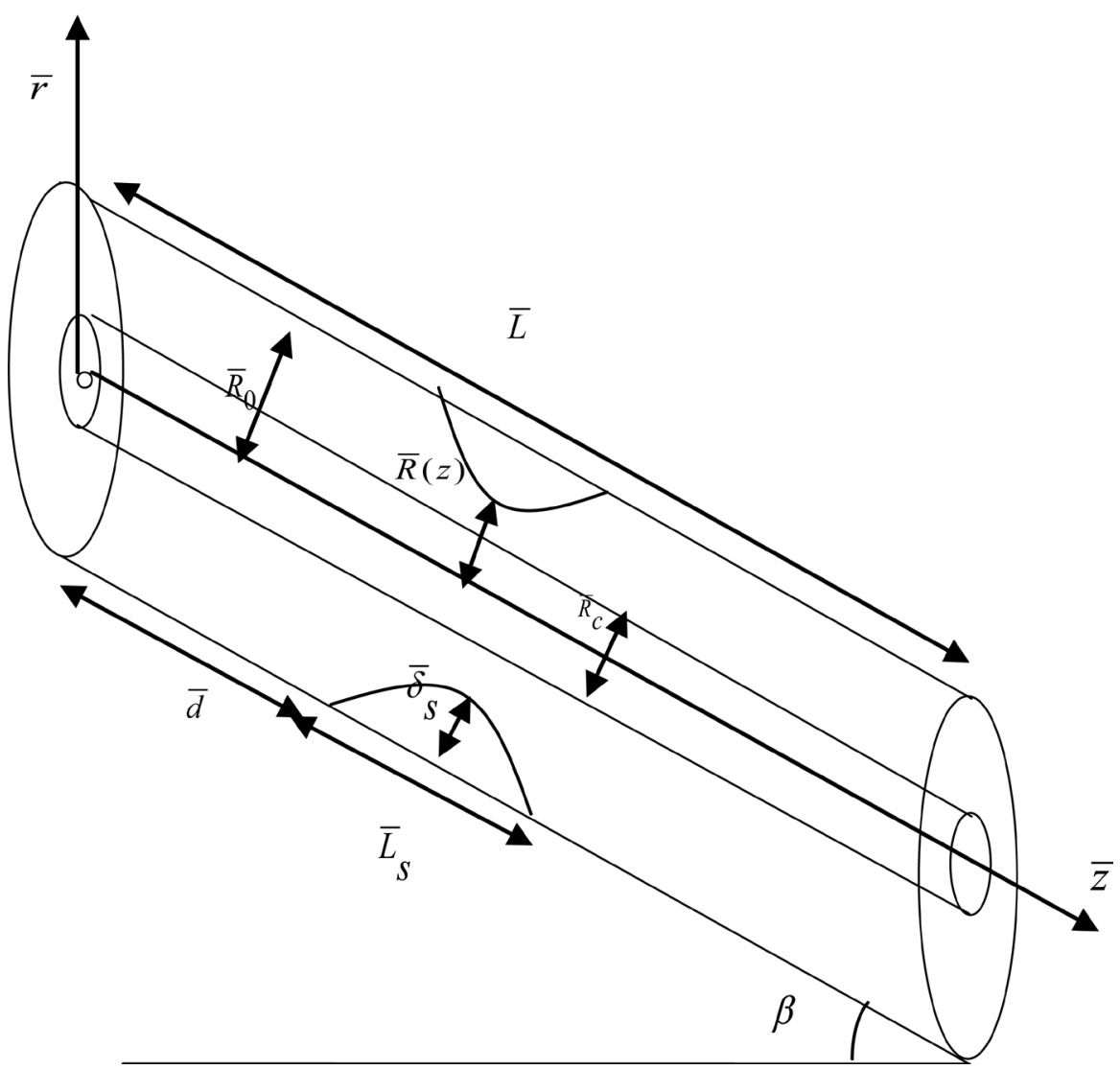

Figure 1. Geometry of an inclined catheterized stenosed artery. 
theterized obstructed artery in the presence of slip and external body acceleration at the arterial wall. The geometry of the stenosis is described in Figure 1. For solving the model we have assumed that blood flow is laminar, incompressible, one dimensional and fully developed in a rigid circular artery of radius $\bar{R}_{0}$ in which a catheter of radius $\bar{R}_{c}$ is introduced coaxially and the blood is modeled as a Newtonian fluid. It is assumed that the pulsatile flow in the artery is due to a prescribed periodic pressure gradient along the axis of the artery. It is also assumed that the length of the artery is large enough when compared to its diameter so that the entrance, end and special wall effects can be neglected.

The geometry of the stenosis which is assumed to be manifested in the arterial segment is described as [26]

$\bar{R}(\bar{z})=\left\{\begin{array}{lr}\bar{R}_{0}-\frac{\bar{\delta}_{s}}{2}\left[1+\frac{\cos 2 \pi}{\bar{L}_{s}}\left(\bar{z}-\bar{d}-\frac{\bar{L}_{s}}{2}\right)\right], \bar{d} \leq \bar{z} \leq \bar{d}+\bar{L}_{s}, \text { the stenosed region } \\ \bar{R}_{0}, & \text { in the normal artery region }\end{array}\right.$

where $\bar{R}(\bar{z})$ and $\bar{R}_{0}$ is the radius of the artery with and without stenosis respectively, $\bar{d}$ is the location of the stenosis, $\bar{L}_{s}$ is the length of the stenosis and $\bar{\delta}_{s}$ denotes the maximum height of the stenosis in to the lumen such that $\bar{\delta}_{s} / \bar{R}_{0} \ll 1$.

We have used cylindrical polar co-ordinates $(\bar{r}, \bar{\phi}, \bar{z})$, whose origin is located on the vessel (stenosed artery) axis and $\bar{r}, \bar{z}$ denote the radial and axial co- ordinates respectively. It can be shown that the magnitude of radial velocity is negligibly small and can be neglected for a low mean Reynold number flow problems in case of mild stenosis.

The Navier-Stokes equations governing the fluid flow is given by, [27].

$$
\begin{gathered}
\bar{\rho}(\partial \bar{v} / \partial \bar{t})=-(\partial \bar{p} / \partial \bar{z})-(1 / \bar{r}) \frac{\partial}{\partial \bar{r}}(\bar{r} \bar{\tau})+\bar{B}(\bar{t})+\bar{\rho} g \sin \beta \\
\partial \bar{p} / \partial \bar{r}=0
\end{gathered}
$$

where $\bar{v}$ represents the axial velocity along $\bar{z}$ direction, $\bar{t}$ is the time, $\bar{\rho}$ is the density, $\bar{p}$ is the pressure, $\bar{\tau}$ is the shear stress, $\beta$ is the inclination angle and $\bar{B}(\bar{t})$ is the body acceleration.

Newtonian fluid can be represented by the equation

$$
\bar{\tau}=-\bar{\mu}(\partial \bar{v} / \partial \bar{r})
$$

where $\bar{\mu}$ denotes the coefficient of viscosity of blood.

The boundary conditions are

$$
\begin{array}{cc}
\bar{v}=\bar{v}_{s} & \text { at } \bar{r}=\bar{R}(\bar{z}) \\
\bar{v}=0 & \text { at } \bar{r}=\bar{R}_{c}
\end{array}
$$

where $\bar{v}_{s}$ is the axial slip velocity at the stenotic wall and $\bar{R}_{c}$ is the radius of catheter.

Since the pressure gradient is the function of $\bar{z}$ and $\bar{t}$, therefore can be represented as $\left(\ll \bar{R}_{0}\right)$. 


$$
\frac{-\partial \bar{p}}{\partial \bar{z}}(\bar{z}, \bar{t})=A_{0}+A_{1} \cos \left(\bar{\omega}_{p} \bar{t}\right), \quad t \geq 0
$$

where $A_{0}$ is the steady state pressure gradient, $A_{1}$ is the amplitude of the fluctuating component and both $A_{0}, A_{1}$ are function of $\bar{z}$. It can be seen that the radial velocity is very small in magnitude so that it may be neglected for problem with mild stenosis. The frequency of oscillation of the pulsatile flow is denoted by $\bar{\omega}_{p}$ and defined as $\bar{\omega}_{p}=2 \pi \bar{f}_{p}$, where $\bar{f}_{p}$ is the pulse rate frequency.

The periodic body acceleration $\bar{B}(\bar{t})$ in the axial direction is given by

$$
\bar{B}(\bar{t})=a_{0} \cos \left(\bar{\omega}_{b} \bar{t}+\phi\right)
$$

where the amplitude of body acceleration is $a_{0}$ and $\phi$ is the phase angle of body acceleration with respect to the pressure gradient. $\bar{\omega}_{b}=2 \pi \bar{f}_{b} ; \bar{f}_{b}$ is its frequency in Hz. The frequency of the body acceleration $\bar{f}_{b}$ is assumed to be small so that wave effect can be neglected.

Let us introduce the following non-dimensional variables

$$
\begin{aligned}
& \left.\begin{array}{l}
v=\frac{\bar{v}}{A_{0} \bar{R}_{0}^{2} / 4 \bar{\mu}}, z=\bar{z} / \bar{R}_{0}, R(z)=\bar{R}(\bar{z}) / \bar{R}_{0}, r=\bar{r} / \bar{R}_{0}, d=\bar{d} / \bar{R}_{0}, \\
L_{s}=\bar{L}_{s} / \bar{R}_{0}, t=\bar{t} \bar{\omega}_{p}, R_{c}=\bar{R}_{c} / \bar{R}_{0}, \delta_{s}=\bar{\delta}_{s} / \bar{R}_{0}, F=A_{0} / 4 \bar{\rho} g, \omega=\bar{\omega}_{b} / \bar{\omega}_{p}, \\
v_{s}=\frac{\bar{v}_{s}}{A_{s} \bar{R}_{0}^{2} / 4 \bar{\mu}}, \tau=\frac{\bar{\tau}}{A_{0} \bar{R}_{0} / 2}, \alpha^{2}=\frac{\bar{R}_{0}^{2} \bar{\omega}_{p} \bar{\rho}}{\bar{\mu}}, e=A_{1} / A_{0}, B=a_{0} / A_{0} .
\end{array}\right\}
\end{aligned}
$$

where $\alpha$ is the pulsatile Reynold's number or generalized Womersley frequency parameter.

Using non-dimensional variables, Equation (2) becomes

$$
\alpha^{2}\left(\frac{\partial v}{\partial t}\right)=4(1+e \cos t)+4 B \cos (\omega t+\phi)-\left(\frac{2}{r}\right) \frac{\partial}{\partial r}(r \tau)+\frac{\sin \beta}{F}
$$

Equation (4) becomes

$$
\tau=-(\partial v / 2 \partial r)
$$

On substituting the value of $\tau$ in Equation (10), we have

$$
\alpha^{2}\left(\frac{\partial v}{\partial t}\right)=4(1+e \cos t)+4 B \cos (\omega t+\phi)+\frac{1}{r} \frac{\partial}{\partial r}\left(r \frac{\partial v}{\partial r}\right)+\frac{\sin \beta}{F}
$$

The boundary conditions (5) and (6) reduces to

$$
\begin{gathered}
v=v_{s} \text { at } r=R(z) \\
v=0 \text { at } r=R_{c}
\end{gathered}
$$

The geometry of an arterial stenosis in non-dimensional form is given by

$R(z)=\left\{\begin{array}{lr}1-\frac{\delta_{s}}{2}\left[1+\frac{\cos 2 \pi}{L_{s}}\left(z-d-\frac{L_{s}}{2}\right)\right], d \leq z \leq d+L_{s}, \text { the stenosed region } \\ 1, \quad \text { in the normal artery region }\end{array}\right.$

The non-dimensional volumetric flow rate is defined by 


$$
Q(z, t)=4 \int_{0}^{R(z)} r v(z, r, t) \mathrm{d} r
$$

where $Q(z, t)=\frac{\bar{Q}(\bar{z}, \bar{t})}{\pi A_{0}\left(\bar{R}_{0}\right)^{4} / 8 \bar{\mu}_{0}} ; \bar{Q}(\bar{z}, \bar{t})$ is the volumetric flow rate.

Effective viscosity $\bar{\mu}_{e}$ defined as

$$
\bar{\mu}_{e}=\pi\left(-\frac{\partial \bar{p}}{\partial z}\right)(\bar{R}(\bar{z}))^{4} / \bar{Q}(\bar{z}, \bar{t})
$$

Can be expressed in the non dimensional form as

$$
\mu_{e}=R^{4}(1+e \cos t) / Q(z, t)
$$

\section{Method of Solution}

In this paper perturbation method is used with a small parameteric value of pulsatile Reynolds number $\alpha$ of the series expansion to solve this system of non linear equations. Since non-dimentionalized Equation (10), Equation (11) has $\alpha^{2}$ term which is dependent on time, therefore expanding Equation (10), Equation (11) about $\alpha^{2}$. The axial velocity $v$, shear stress $\tau$ are expressed as follows in terms of $\alpha^{2}$ (where $\left.\alpha^{2} \ll 0\right)$.

$$
\begin{gathered}
v(z, r, t)=v_{0}(z, r, t)+\alpha^{2} v_{1}(z, r, t)+\cdots \\
\tau(z, r, t)=\tau_{0}(z, r, t)+\alpha^{2} \tau_{1}(z, r, t)+\cdots
\end{gathered}
$$

Substituting Equation (18) in Equation (12), we get

$$
\begin{gathered}
\frac{1}{r} \frac{\partial}{\partial r}\left(r \frac{\partial v_{0}}{\partial r}\right)=-4 r h(t) \\
\frac{\partial v_{0}}{\partial t}=\frac{1}{r} \frac{\partial}{\partial r}\left(r \frac{\partial v_{1}}{\partial r}\right)
\end{gathered}
$$

where $h(t)=\left[(1+e \cos t)+B \cos (\omega t+\phi)+\frac{\sin \beta}{4 F}\right]$

Substituting Equation (18) in Equation ((12) and (13)), we have

$$
\begin{array}{cc}
v_{0}=v_{s}, v_{1}=0 \quad \text { at } r=R(z) \\
v_{0}=0, v_{1}=0 \quad \text { at } r=R_{c}
\end{array}
$$

Integrating Equations ((20) and (21)), with the help of Equations ((22) and (23)), the expression for $v_{0}$ and $v_{1}$ are obtained as

$$
\begin{gathered}
v_{0}=v_{s}\left(1+\frac{\log _{e}(r / R)}{\log _{e}\left(R / R_{c}\right)}\right)+h(t)\left\{\left(R^{2}-r^{2}\right)+\left(R^{2}-R_{c}^{2}\right) \frac{\log _{e}(r / R)}{\log _{e}\left(R / R_{c}\right)}\right\} \\
v_{1}=H(t)\left[2 r^{2}\left\{\left(R^{2}-\frac{r^{2}}{4}\right)+\left(R^{2}-R_{c}^{2}\right) \frac{\log _{e}(r / R)}{\log _{e}\left(R / R_{c}\right)}\right\}-\frac{\left(R^{2}-R_{c}^{2}\right)}{\log _{e}\left(R / R_{c}\right)}\left(R^{2}+r^{2}\right)\right. \\
\left.-\frac{3 R^{4}}{2}+\frac{\log _{e}(r / R)}{\log _{e}\left(R / R_{c}\right)}\left\{\frac{\left(R^{4}+R_{c}^{4}\right)}{\log _{e}\left(R / R_{c}\right)}-\frac{2 R^{2} R_{c}^{2}}{\log _{e}\left(R / R_{c}\right)}-2\left(R^{4}-R_{c}^{4}\right)\right\}\right]
\end{gathered}
$$


where $H(t)=h^{\prime}(t) / 8$

With the help of Equations ((18), (24) and (25)), the expression for the axial velocity $v$ can be obtained as

$$
\begin{aligned}
v= & v_{s}\left(1+\frac{\log _{e}(r / R)}{\log _{e}\left(R / R_{c}\right)}\right)+h(t)\left\{\left(R^{2}-r^{2}\right)+\left(R^{2}-R_{c}^{2}\right) \frac{\log _{e}(r / R)}{\log _{e}\left(R / R_{c}\right)}\right\} \\
& +\alpha^{2} H(t)\left[2 r^{2}\left\{\left(R^{2}-\frac{r^{2}}{4}\right)+\left(R^{2}-R_{c}^{2}\right) \frac{\log _{e}(r / R)}{\log _{e}\left(R / R_{c}\right)}\right\}-\frac{\left(R^{2}-R_{c}^{2}\right)}{\log _{e}\left(R / R_{c}\right)}\left(R^{2}+r^{2}\right)\right. \\
& \left.-\frac{3 R^{4}}{2}+\frac{\log _{e}(r / R)}{\log _{e}\left(R / R_{c}\right)}\left\{\frac{\left(R^{4}+R_{c}^{4}\right)}{\log _{e}\left(R / R_{c}\right)}-\frac{2 R^{2} R_{c}^{2}}{\log _{e}\left(R / R_{c}\right)}-2\left(R^{4}-R_{c}^{4}\right)\right\}\right]
\end{aligned}
$$

With the help of Equations ((11) and (19)), the wall shear stress $\tau_{w}$ can be obtained as

$$
\begin{gathered}
\tau_{w}=-\frac{1}{2}\left\{\left(\frac{\partial v_{0}}{\partial r}\right)+\alpha^{2}\left(\frac{\partial v_{1}}{\partial r}\right)\right\}_{r=R(z)} \\
\tau_{w}=\left\{R h(t)-\frac{\left(h(t)\left(R^{2}-R_{c}^{2}\right)+v_{s}\right)}{2 R \log _{e}\left(R / R_{c}\right)}\right\}-\alpha^{2} H(t) \\
\cdot\left[R^{3}-\frac{1}{R \log _{e}\left(R / R_{c}\right)}\left\{\left(R^{4}-R_{c}^{4}\right)+\frac{R_{c}^{2} R^{2}}{\log _{e}\left(R / R_{c}\right)}-\frac{\left(R^{4}+R_{c}^{4}\right)}{2 \log _{e}\left(R / R_{c}\right)}\right\}\right]
\end{gathered}
$$

From Equations ((16) and (26)) the expression for volumetric flow rate $Q(z, t)$ can be obtained as

$$
\begin{aligned}
& Q(z, t)=h(t)\left\{R^{4}-\frac{R^{2}\left(R^{2}-R_{c}^{2}\right)}{4 \log _{e}\left(R / R_{c}\right)}\right\}+v_{s}\left\{2 R^{2}-\frac{R^{2}}{\log _{e}\left(R / R_{c}\right)}\right\}+\frac{\alpha^{2} H(t)}{2} \\
& \cdot\left[\frac{R^{4}\left(R^{2}-R_{c}^{2}\right)}{\log _{e}\left(R / R_{c}\right)}-2 R^{6}-\frac{R^{2}}{\log _{e}\left(R / R_{c}\right)}\left\{\frac{2\left(R^{4}+R_{c}^{4}\right)}{\log _{e}\left(R / R_{c}\right)}-4\left(R^{4}-R_{c}^{4}\right)-\frac{4 R^{2} \cdot R_{c}^{2}}{\log _{e}\left(R / R_{c}\right)}\right\}\right]
\end{aligned}
$$

The expression for effective viscosity $\mu_{e}$ can be obtained from Equationd ((17) and (26)) as

$$
\begin{aligned}
& \mu_{e}=R^{4}(1+e \cos t) \cdot\left[h(t)\left\{R^{4}-\frac{R^{2}\left(R^{2}-R_{c}^{2}\right)}{4 \log _{e}\left(R / R_{c}\right)}\right\}+v_{s}\left\{2 R^{2}-\frac{R^{2}}{\log _{e}\left(R / R_{c}\right)}\right\}+\frac{\alpha^{2} H(t)}{2}\right. \\
& \left.\cdot\left[\frac{R^{4}\left(R^{2}-R_{c}^{2}\right)}{\log _{e}\left(R / R_{c}\right)}-2 R^{6}-\frac{R^{2}}{\log _{e}\left(R / R_{c}\right)} \cdot\left\{\frac{2\left(R^{4}+R_{c}^{4}\right)}{\log _{e}\left(R / R_{c}\right)}-4\left(R^{4}-R_{c}^{4}\right)-\frac{4 R^{2} \cdot R_{c}^{2}}{\log _{e}\left(R / R_{c}\right)}\right\}\right]\right]^{-1}
\end{aligned}
$$

\section{Numerical Results and Discussion}

In the present investigation our objective is, to carry out the combined result of applied body acceleration and slip velocity in an inclined catheterized stenosed artery. The expression of axial velocity, flow rate, wall shear stress and effective viscosity are obtained and computed for the fixed values of $F=0.2, e=1, \phi=$ 
$0.2, \alpha=0.5, \omega=1$ through the MATLAB. Figures 2-5 reveals the variation of axial velocity with radial distance. Figure 2 depicts that the magnitude of axial velocity increases on increasing slip velocity and almost double for increased value of body acceleration from $B=0$ to $B=2$. From Figure 3 and Figure 4, we observed that the axial velocity is greater in inclined artery along with the increase in slip as compared to the normal artery (non-inclined) without slip and body acceleration also enhances the velocity graph in inclined position.

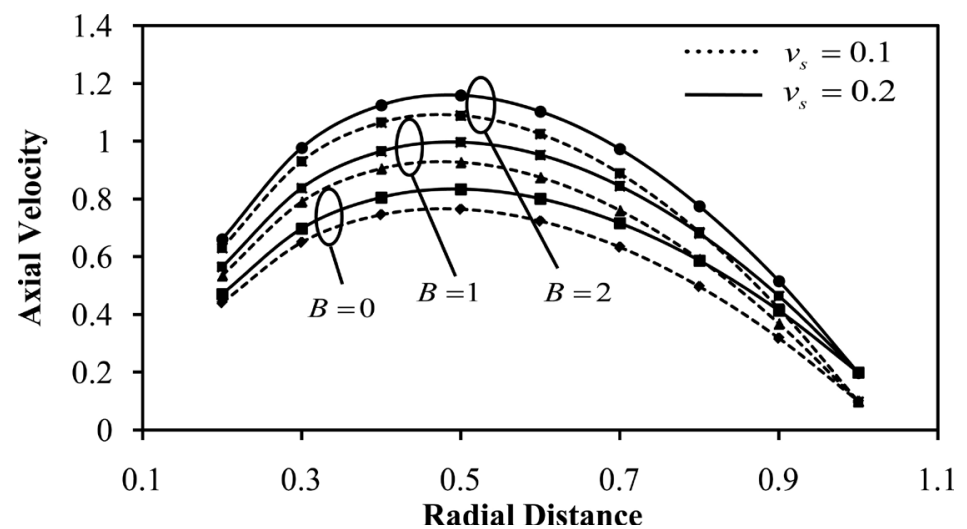

Figure 2. Variation of axial velocity with the radial distance at $\alpha=0.1, e=1, \delta=0.1, \beta=0$.

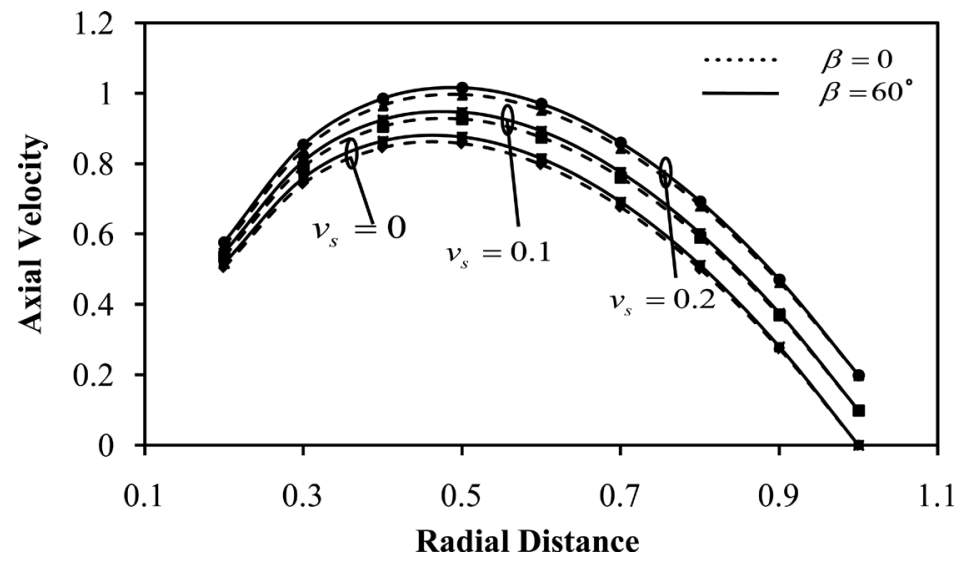

Figure 3. Variation of axial velocity with the radial distance at $B=1, \mathrm{e}=1, \alpha=0.1, \delta=0.1$.

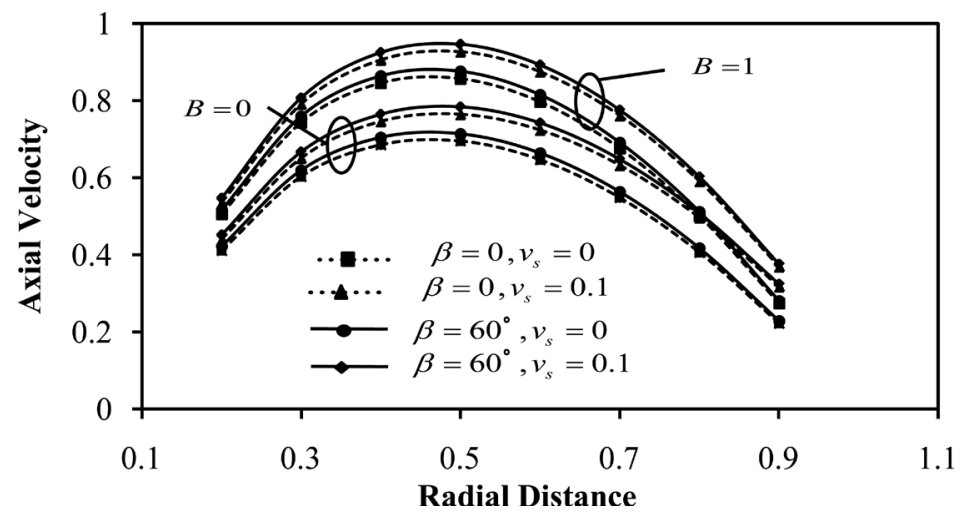

Figure 4. Variation of axial velocity with the radial distance at $B=1, \alpha=0.1, e=1, \delta=$ $0.1, t=1$. 
Figure 5 shows that on increasing the catheter radius, axial velocity decreases while slip improves the velocity profile. In Figure 6 and Figure 7, we dealt with flow rate versus axial distance and we found that flow rate increases with the increase in body acceleration, slip. Flow rate slightly increases when artery is inclined and perpendicular to the horizontal axis as compared to the normal (non-inclined) artery. The variation of wall shear stress with axial distance is

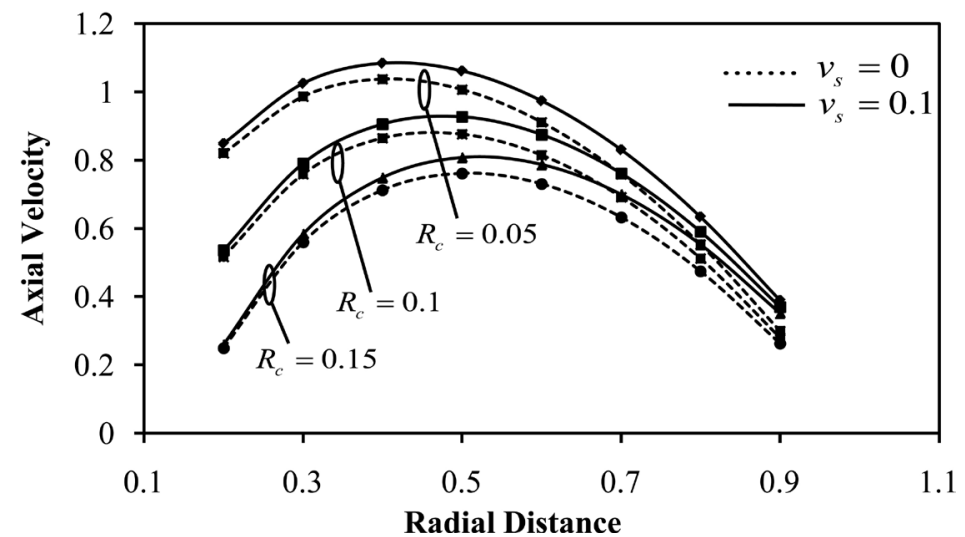

Figure 5. Variation of axial velocity with the radial distance at $B=1, \alpha=0.1, e=1, \delta=$ $0.1, t=1$.

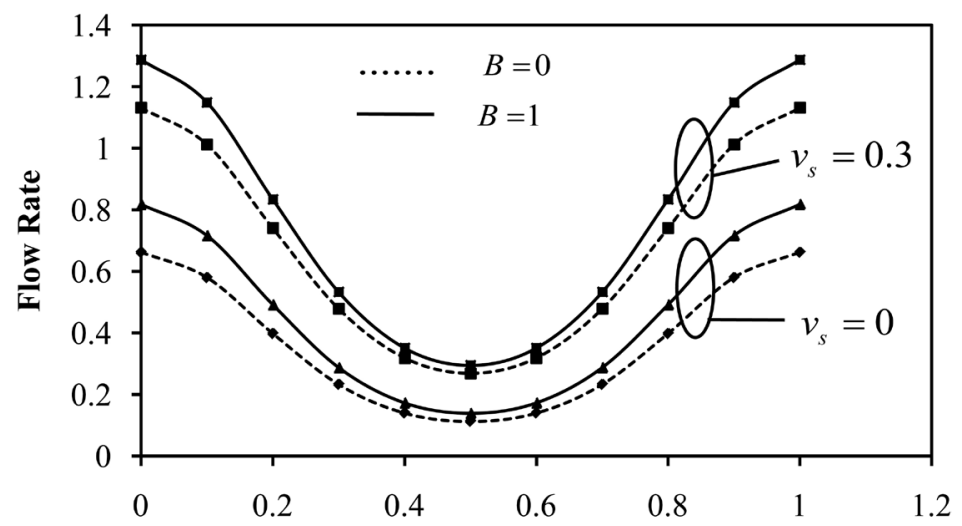

Axial Distance

Figure 6. Variation of flow rate with the axial distance at $\alpha=0.1, e=1, \delta=0.1$.

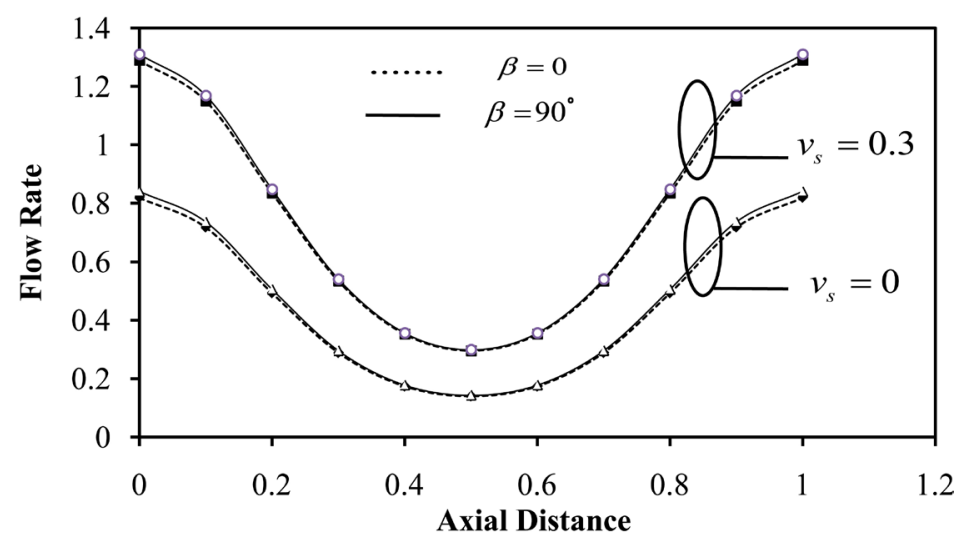

Figure 7. Variation of flow rate with the axial distance at $B=1, \alpha=0.1, e=1, \delta=0.1, t=1$. 
observed in Figure 8, which depicts that the wall shear stress increases with the increase in catheter radius. Figure 9, wall shear stress versus catheter radius shows that increase in catheter radius and body acceleration increases the wall shear stress. Also increasing slip velocity, reduces the wall shear stress can be easily noticed in Figure 8 and Figure 9. Effective viscosity with stenosis height

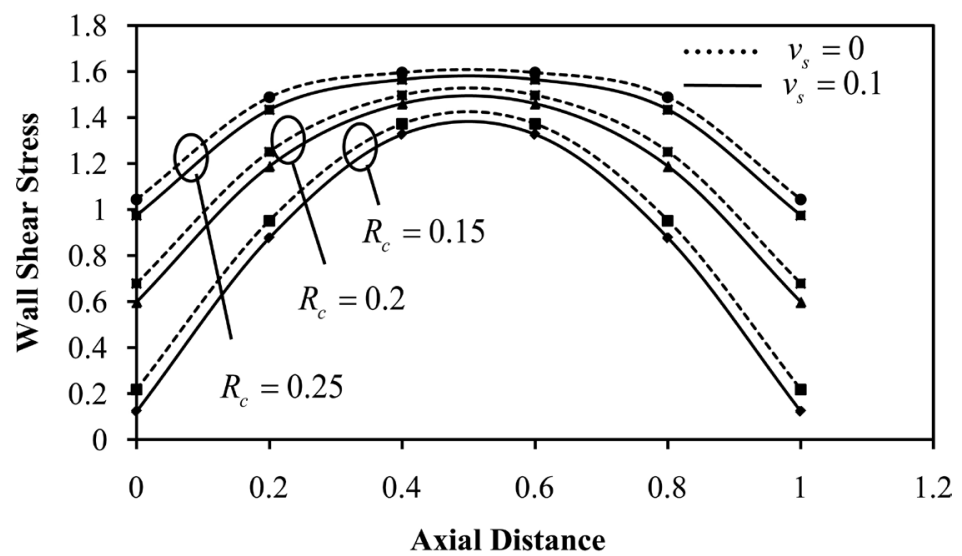

Figure 8. Variation of wall shear stress with the axial distance at $B=1, \alpha=0.1, e=1, \delta=$ $0.1, t=1$.

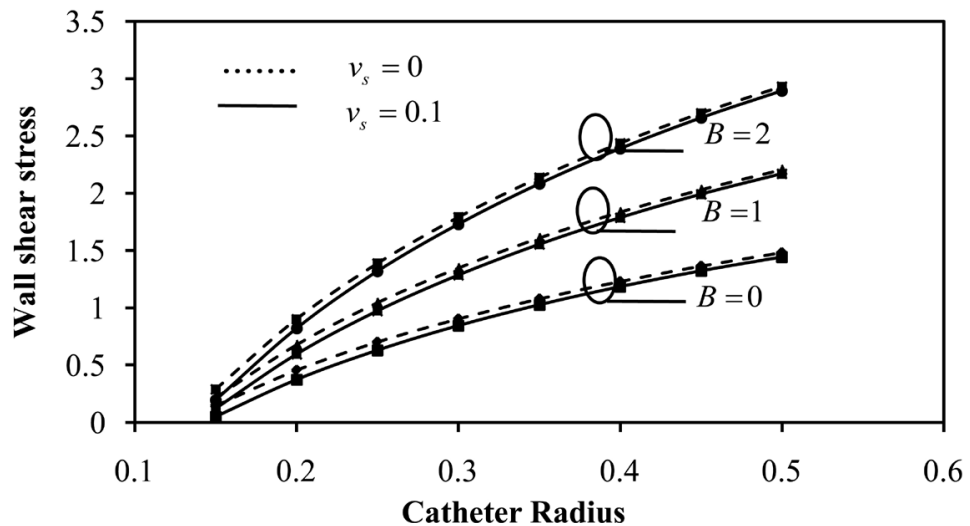

Figure 9. Variation of wall shear stress with cathter radius at $\alpha=0.1, e=1, \delta=0.1$.

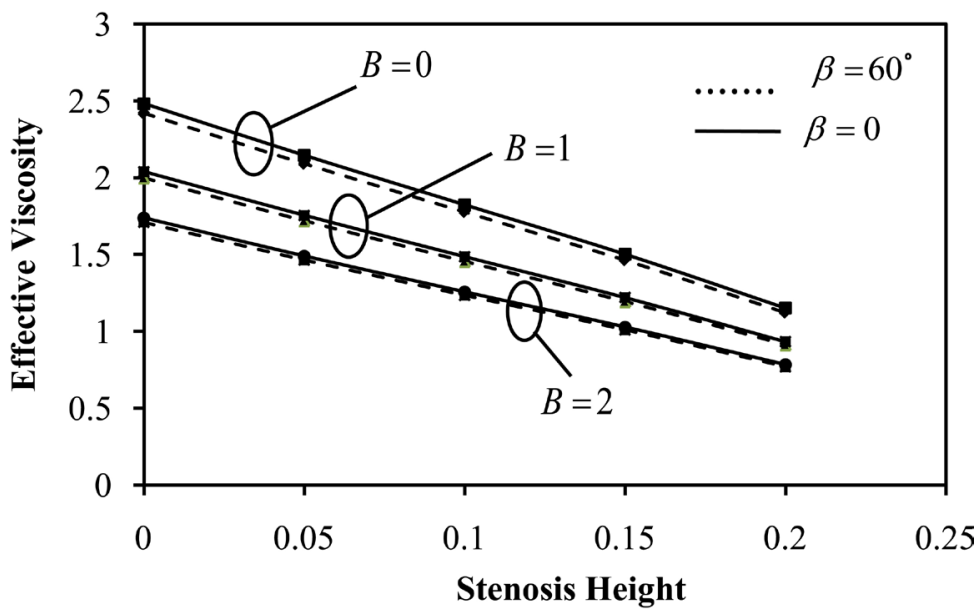

Figure 10. Variation of effective viscosity with the stenosis height at $\alpha=0.1, e=1, \delta=0.1$. 
for different values of body acceleration has been seen in Figure 10 and found that on increasing body acceleration and inclination angle, effective viscosity diminishes whereas slightly augmented in normal (non-inclined) stenosed artery.

\section{Conclusion}

In the present mathematical model, pulsatile blood flow through an inclined stenosed catheterized artery with periodic body acceleration and axial slip velocity at the constricted wall has been considered. The flowing fluid is represented by a Newtonian fluid. Analytic expressions for flow variables and their variations with different flow parameters have been obtained and are represented graphically. The results based on the mathematical analysis and the subsequent numerical evaluation of the flow quantities show that the axial velocity and flow rate increases with the increase in body acceleration, inclination angle and slip velocity while axial velocity diminishes on increasing the catheter radius. Wall shear stress increases with the increase in catheter radius and body acceleration but presence of slip velocity reduces the wall shear stress. Effective viscosity diminishes on increasing body acceleration and inclination angle, whereas slightly augmented in normal (non-inclined) stenosed artery. This model concludes that slip velocity plays a very eminent role in blood flow models through an inclined stenosed artery with catheter. In future, this study may be helpful for the purpose of simulation and validation of different models in different conditions of stenosis and to investigate that which parameter has the most dominating role.

\section{Acknowledgements}

Corresponding author (Chhama Awasthi) is thankful to the TEQIP (SPFU, World Bank) at HBTU, Kanpur, India for awarding Fellow-ship for this research work.

\section{References}

[1] Bennett, L. (1967) Red Cell Slip at a Wall in Vitro. Science, 155, 1554-1556. https://doi.org/10.1126/science.155.3769.1554

[2] Tu, C. and Deville, M. (1996) Pulsatile Flow of Non-Newtonian Fluids through Arterial Stenosis. Journal of Biomechanic, 29, 899-908. https://doi.org/10.1016/0021-9290(95)00151-4

[3] Misra, J.C. and Shit, G.C. (2007) Role of Slip Velocity in Blood Flow through Stenosed Arteries: A Non-Newtonian Model. Journal of Mechanics in Medicine and Biology, 7, 337-353. https://doi.org/10.1142/S0219519407002303

[4] Ponalgusamy, R. (2007) Blood Flow through an Artery with Mild Stenosis: A Two-Layered Model, Different Shapes of Stenoses and Slip Velocity at the Wall. Journal of Applied Sciences, 7, 1071-1077. https://doi.org/10.3923/jas.2007.1071.1077

[5] Sud, V.K. and Sekhon, G.S. (1985) Arterial Flow under Periodic Body Acceleration. Bulletin of Mathematical Biology, 47, 35-52. https://doi.org/10.1007/BF02459645 
[6] Chakravarthy, S. and Mandal, P.K. (2000) Two-Dimensional Blood Flow through Tapered Arteries under Stenotic Conditions. International Journal Non-Linear Mechanics, 35, 779-793. https://doi.org/10.1016/S0020-7462(99)00059-1

[7] Liu, G.T., Wang, X.J., Ai, B.Q. and Liu, L.G. (2004) Numerical Study of Pulsating Flow through a Tapered Artery with Stenosis. China Journal Physics, 42, 401-409.

[8] Young, D.F. (1968) Effects of Time Dependent Stenosis on Flow through a Tube. Journal of Engineering and Industrial Transactions, ASME, 90, 248-254. https://doi.org/10.1115/1.3604621

[9] Liepsch, D.W. (1986) Flow in Tubes and Arteries-A Comparison. Biorheology, 23, 395-433.

[10] Young, D.F. and Tsai, F.Y. (1973) Flow Characteristics in Models of Arterial Stenosis-Steady Flow. Journal of Biomechanics, 6, 395-411. https://doi.org/10.1016/0021-9290(73)90099-7

[11] Mishra, J.C., Patra, M.K. and Mishra, S.C. (1993) A Non-Newtonian Fluid Model for Blood Flow through Arteries under Stenotic Conditions. Journal of Biomechanics, 26, 1129-1141.

[12] Ookawara, S. and Ogowa, K. (2000) Flow Properties of Newtonian and Non Newtonian Fluid Downstream of Stenosis. Journal of Chemical Engineering Japan, 33, 582-590. https://doi.org/10.1252/jcej.33.582

[13] Srinivasacharya, D. and Srikanth, D. (2008) Effect of Couple Stresses on the Flow in a Constricted Annulus. Archive of Applied Mechanics, 78, 251-257. https://doi.org/10.1007/s00419-007-0157-6

[14] MacDonald, D.A. (1986) Pulsatile Flow in a Catheterised Artery. Journal of Biomechanics, 19, 239-249.

[15] Karahalios, G.T. (1990) Some Possible Effects of a Catheter on the Arterial Wall. Medical Physics, 17, 902-925. https://doi.org/10.1118/1.596448

[16] Back, L.H. and Denton, T.A (1992) Some Arterial Wall Shear Stress Estimates in Coronary Angioplasty. Advanced Biomedical Engineering, 22, 337-340.

[17] Kanai, H., Lizuka, M. and Sakamotos, K. (1970) One of the Problem in the Measurement of Blood Pressure by Catheterization; Wave Reflection at the Tip of Catheter. Medical Biological Engineering, 28, 483-496.

https://doi.org/10.1007/BF02477185

[18] Sarkar, A. and Jayaraman, G. (1998) Correction to Flow Rate-Pressure Drop in Coronary Angioplasty: Steady Streaming Effect. Journal of Biomechanics, 31, 781-791.

[19] Verma, N.K., Mishra, S., Siddiqui, S.U. and Gupta, R.S. (2011) Effect of Slip Velocity on Blood Flow through Catheterized Artery. Applied Mathematics, 2, 764-770. https://doi.org/10.4236/am.2011.26102

[20] Chaturani, P. and Upadhya, V.S. (1977) Gravity Flow of Fluid with Couple Stress along an Inclined Plane with Application to Blood Flow. Journal of Biorheology, 14, 237-246.

[21] Vajravelu, K., Sreenadh, S. and Ramesh Babu, V. (2005) Peristaltic Transport of a Herschel Bulkley Fluid in an Inclined Tube. International Journal of Non-Linear Mechanics, 40, 83-90.

[22] Rathod, V.P., Begum, N. and Sheeba, R.I. (2006) Inclined Pulsatile Flow of Blood with Periodic Body Acceleration. Acta Ciencia Indica, 32, 1509-1516.

[23] Maruti Prasad, K. and Radhakrishnamacharya, G. (2008) Flow of Herschel-Bulkley Fluid through an Inclined Tube of Non-Uniform Cross Section with Multiple Stenosis. Archives of Mechanics, 60, 161-172. 
[24] Biswas, D. and Paul, M. (2013) Study of Blood Flow Inside an Inclined Non-Uniform Stenosed Artery. International Journal of Mathematical Archive, 4, 33-42.

[25] Sharma, M.K., Singh, K. and Bansal, S. (2014) Pulsatile MHD Flow in an Inclined Catheterized Stenosed Artery with Slip on the Wall. Journal of Biomedical Sciences and Engineering, 7, 194-207. https://doi.org/10.4236/jbise.2014.74023

[26] Sankar, D.S. and Hemalatha, K. (2006) Pulsatile Flow of Herschel Bulkey Fluid through Stenosed Arteries-A Mathematical Model. International Journal of Non-Linear mechanics, 41, 979-990.

[27] Schlichting, H. and Gersten, K. (2004) Boundary Layer Theory. Springer-Verlag.

Submit or recommend next manuscript to SCIRP and we will provide best service for you:

Accepting pre-submission inquiries through Email, Facebook, LinkedIn, Twitter, etc. A wide selection of journals (inclusive of 9 subjects, more than 200 journals)

Providing 24-hour high-quality service

User-friendly online submission system

Fair and swift peer-review system

Efficient typesetting and proofreading procedure

Display of the result of downloads and visits, as well as the number of cited articles

Maximum dissemination of your research work

Submit your manuscript at: http://papersubmission.scirp.org/

Or contact jamp@scirp.org 